\title{
Altering thermal transport by strained-layer epitaxy
}

Tahereh Majdi, Souvik Pal, Anders Hafreager, Sohail Murad, Rakesh P. Sahu, and Ishwar K. Puri

Citation: Appl. Phys. Lett. 112, 194101 (2018); doi: 10.1063/1.5022097

View online: https://doi.org/10.1063/1.5022097

View Table of Contents: http://aip.scitation.org/toc/apl/112/19

Published by the American Institute of Physics

\section{Articles you may be interested in}

Acoustic phonon spectrum engineering in bulk crystals via incorporation of dopant atoms

Applied Physics Letters 112, 191902 (2018); 10.1063/1.5030558

Temporal-spatial measurement of electron relaxation time in femtosecond laser induced plasma using two-color pump-probe imaging technique

Applied Physics Letters 112, 191101 (2018); 10.1063/1.5009062

Energy and charge transfer effects in two-dimensional van der Waals hybrid nanostructures on periodic gold nanopost array

Applied Physics Letters 112, 193101 (2018); 10.1063/1.5023997

Mimicking graphene physics with a plane hexagonal wire mesh

Applied Physics Letters 112, 191601 (2018); 10.1063/1.5026355

Room temperature ferromagnetism in transition metal-doped black phosphorous

Applied Physics Letters 112, 192105 (2018); 10.1063/1.5022540

Quantitative study of bundle size effect on thermal conductivity of single-walled carbon nanotubes

Applied Physics Letters 112, 191904 (2018); 10.1063/1.5021696

\section{Conference Proceedings}




\title{
Altering thermal transport by strained-layer epitaxy
}

\author{
Tahereh Majdi, ${ }^{1}$ Souvik Pal, ${ }^{2}$ Anders Hafreager, ${ }^{3}$ Sohail Murad, ${ }^{4}$ Rakesh P. Sahu, ${ }^{5}$ \\ and Ishwar K. Puri ${ }^{1,5, a)}$ \\ ${ }^{1}$ Department of Engineering Physics, McMaster University, Hamilton, Ontario L8S 4L7, Canada \\ ${ }^{2}$ Computing Infrastructure Research Center, McMaster University, Hamilton, Ontario L8P 0A1, Canada \\ ${ }^{3}$ Department of Physics, University of Oslo, P.O. Box 1033, Blindern, N-0315 Oslo, Norway \\ ${ }^{4}$ Department of Chemical and Biological Engineering, Illinois Institute of Technology, Chicago, \\ Illinois 60616, USA \\ ${ }^{5}$ Department of Mechanical Engineering, McMaster University, Hamilton, Ontario L8S 4L7, Canada
}

(Received 10 January 2018; accepted 16 April 2018; published online 8 May 2018)

\begin{abstract}
Since strain changes the interatomic spacing of matter and alters electron and phonon dispersion, an applied strain can modify the thermal conductivity $k$ of a material. We show how the strain induced by heteroepitaxy is a passive mechanism to change $k$ in a thin film. Molecular dynamics simulations of the deposition and epitaxial growth of ZnTe thin films provide insights into the role of interfacial strain in the conductivity of a deposited film. ZnTe films grow strain-free on latticematched ZnTe substrates, but similar thin films grown on a lattice-mismatched CdTe substrate exhibit $\sim 6 \%$ biaxial in-plane tensile strain and $\sim 7 \%$ uniaxial out-of-plane compressive strain. In the $T=700 \mathrm{~K}-1100 \mathrm{~K}$ temperature range, the conductivities of strained $\mathrm{ZnTe}$ layers decrease to $\sim 60 \%$ of their unstrained values. The resulting understanding of $\mathrm{d} k / \mathrm{d} T$ shows that strain engineering can be used to alter the performance of a thermal rectifier and also provides a framework for enhancing thermoelectric devices. Published by AIP Publishing. https://doi.org/10.1063/1.5022097
\end{abstract}

It is possible to alter a material's thermal conductivity $k$ through passive and nondestructive mechanisms. Altering $k$ can be useful to modulate heat dissipation in integrated electronics or for thermoelectrics that require low $k$ but high electrical conductivity. ${ }^{1-4}$ Furthermore, tuning $\mathrm{d} k / \mathrm{d} T$ is relevant in designing thermal rectifiers, where the heat flux depends on the direction of the temperature gradient. ${ }^{5,6} \mathrm{We}$ utilize the strain induced by heteroepitaxy as a passive method to alter $k$ in a thin film. Since this mechanism of strain induction can be realized in practice, it has practical relevance for device design. Previous experiments indicate that $\mathrm{SrTiO}_{3} / \mathrm{CaTiO}_{3}$ superlattices grown on $\mathrm{NdGaO}_{3}$ have a larger thermal conductivity than when the superlattices are grown on $\mathrm{SrTiO}_{3}$ substrates. The measured difference in $k$ is attributed to the differences in-plane strains of $-0.1 \%$ and $0.93 \%$ that are produced for the two cases, respectively. ${ }^{7,8}$ Our systematic simulations unambiguously quantify the effect of heteroepitaxial strain on $k$. Unlike the existing heat transfer literature for lattice mismatched heterostructures, ${ }^{9,10}$ this entry simulates the complete cycle of thin film deposition and growth, evaluates the deposited film's thermal conductivity, and probes the applicability of epitaxy-induced strain for a thermal rectifying device.

Molecular Dynamics (MD) simulations are employed to investigate the deposition and growth of thin films ${ }^{11}$ and their thermal responses. ${ }^{12,13}$ The atomic coordinates of the substrate are specified a priori. As the constituent atoms are deposited, an epitaxial thin film grows with a crystallographic orientation that is influenced by the substrate. During homoepitaxy, both the thin film and the substrate are composed of the same elements so that the film-substrate lattice parameters are perfectly matched. Hence, the material

\footnotetext{
${ }^{\text {a) }}$ Author to whom correspondence should be addressed: ikpuri@mcmaster.ca. Tel.: +1 (905) 525-9140 ext. 24900.
}

does not experience interfacial-bond straining. In the case of heteroepitaxy, the film and substrate materials are different and thus have mismatched lattice parameters. Depending on the deposition conditions, the extent of lattice mismatch, and the thickness of the deposited film, the interfacial adatoms of the film can become either strained or relaxed by forming dislocation defects at the interface. ${ }^{14}$

Specifically, we consider strained-layer epitaxy in ZnTe-CdTe heterostructures, with both materials being wide bandgap II-VI semiconductors used in optoelectronic devices. ${ }^{15,16}$ Their heterostructures have a lattice mismatch of $\left(\mathrm{a}_{s u}-\mathrm{a}_{f}\right) / \mathrm{a}_{f} \approx 6 \%$, where the subscripts $s u$ and $f$ denote the substrate and the thin film, respectively. This mismatch is small enough to allow high quality crystal growth but sufficiently large to unambiguously reveal changes in $k$. Hence, these materials provide an ideal case to explore the influence of strained-layer epitaxy on $k$. For reference, we also simulate the homoepitaxial growth of relaxed ZnTe films and compare their thermal responses with those of the strained heteroepitaxial ZnTe films grown on CdTe substrates. Both the homoepitaxial and heteroepitaxial grown films are dislocation free. Nonequilibrium MD studies provide $k$ for the ZnTe films, which are evaluated between 700 and $1100 \mathrm{~K}$. These temperatures lie well below the melting points of ZnTe $(1568 \mathrm{~K})$ and CdTe $(1315 \mathrm{~K})^{17}$ and exceed their Debye temperature values $\theta_{\mathrm{D}}$, i.e., $\mathrm{ZnTe}(225 \mathrm{~K})$ and $\mathrm{CdTe}$ $(158 \mathrm{~K}),{ }^{18}$ ensuring that the MD simulations are relevant.

The simulations are conducted using LAMMPS $^{19}$ and employ the Zn-Cd-Hg-S-Se-Te Stillinger-Weber (SW) potential that appropriately reproduces experimental lattice constants, cohesive energies, and elastic constants, while ensuring that the lowest energy structure is tetrahedral. ${ }^{20}$ Stillinger-Weber potentials ${ }^{21}$ have a term in their potential energy function that penalizes non-tetrahedral bond formation, an important property that allows the potential to predict the 


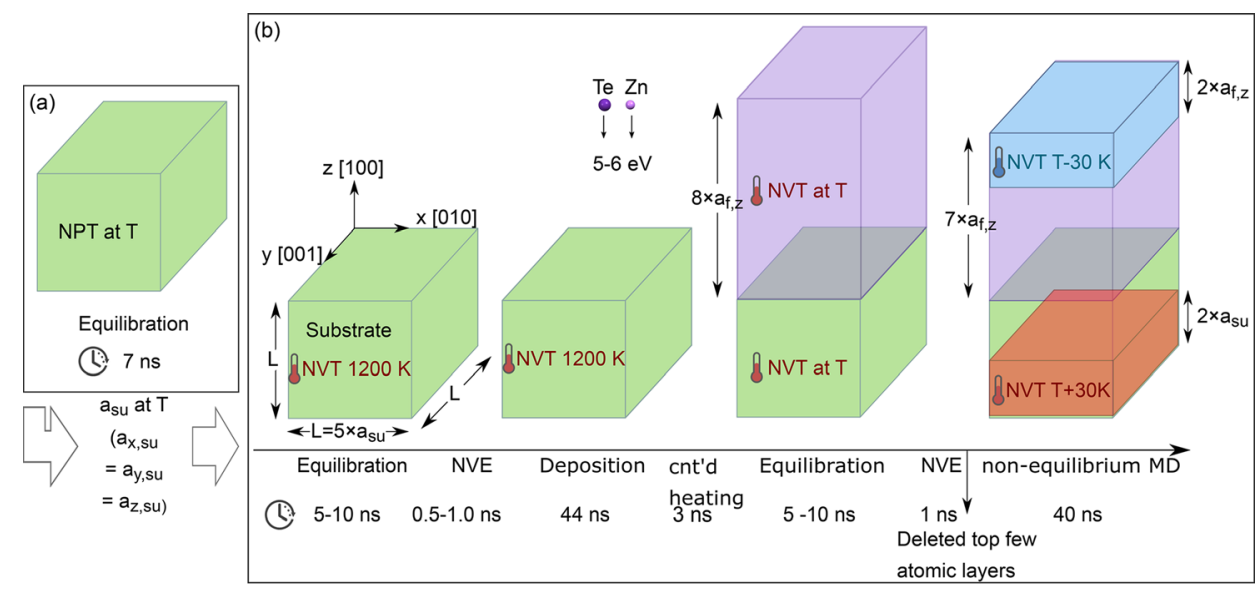

FIG. 1. Simulation overview. (a) Simulations are conducted using periodic boundary conditions, under NPT, to determine the equilibrium lattice constant. The substrate is a Zincblende crystal, either Zn-terminated (upper most surface) ZnTe or Cd-terminated CdTe. The calculated lattice constants are used as inputs into subsequent simulations shown in (b). $a_{s u}$ and $a_{f, z}$ denote the substrate and thin film lattice constants along the z-direction. For homoepitaxy, $a_{f, z}=a_{s u}$, and for heteroepitaxy, with CdTe as the substrate, $a_{f, z}<a_{s u}$. The $x$ and $y$ boundaries in (b) are periodic, while the $z$ boundary is fixed. crystalline growth of stoichiometric compounds under stoichiometric growth conditions during MD simulations of vapor deposition. $^{20}$ To evaluate the suitability of this potential for examining the thermal conductivity of ZnTe, a series of simulations are conducted, the results from which are averaged over $50 \mathrm{~ns}$, to extrapolate the bulk thermal conductivity of $\mathrm{ZnTe}$ at $300 \mathrm{~K} .^{22}$ The detailed methodology is described in supplementary material A. The extrapolated thermal conductivity for a cross-sectional area of $4 \times 4$ [(unit cells $)^{2}$ ] is $19 \pm 1 \mathrm{~W} \mathrm{~m}^{-1} \mathrm{~K}^{-1}$, and the corresponding value for $12 \times 12$ $\left[(\text { unit cells) })^{2}\right.$ is $18 \pm 2 \mathrm{~W} \mathrm{~m}^{-1} \mathrm{~K}^{-1}$, which are both in agreement with the experimentally measured value of $18 \mathrm{~W} \mathrm{~m}^{-1} \mathrm{~K}^{-1}$ for ZnTe. ${ }^{18}$

An overview of the MD simulations is presented in Fig. 1. First, as shown in Fig. 1(a), simulations are performed with a 0.4 fs time step under constant NPT-number of atoms, pressure, and temperature-with periodic boundaries to determine the equilibrium lattice constant of the substrate between 700 and $1100 \mathrm{~K}$. The substrate is either ZnTe or CdTe with a Zincblende crystal structure. In this temperature range, the equilibrium lattice constant of $\mathrm{CdTe}$ ranges from 6.507 to $6.525 \AA$ and for ZnTe from 6.133 to $6.155 \AA$. For reference, lattice constants for both materials are also evaluated at $293 \mathrm{~K}$ and found to be only $0.1 \%$ larger than experimental measurements at $\sim 300 \mathrm{~K}$ with a lattice mismatch that is in agreement with experiments. The slight overestimate of the lattice parameters is characteristic of the potential function employed. ${ }^{11,23}$ Table S1 of supplementary material A contains a full list of the measured lattice constants.

Subsequent simulations, presented in Fig. 1(b), use these values for the lattice constants. The periodic $x$ and $y$ boundaries are 5 unit cells wide, the $z$ boundary is fixed and 18 unit cells high, and the bottommost layer is fixed and immobile. The substrate is equilibrated to $1200 \mathrm{~K}$ for $5-10 \mathrm{~ns}$ under constant NVT-number of atoms, volume, and temperature-using Nosé-Hoover style thermostats. Substrate thermostatting continues until 3 ns after deposition, whereupon $1 \mathrm{Zn}$ and $1 \mathrm{Te}$ atom are introduced from the top of the simulation box every $55 \mathrm{ps}$ with an incident angle of $0^{\circ}$ and an incident energy of $5.3 \mathrm{eV}-6 \mathrm{eV}$. Following deposition, the deposited thin film and the substrate are equilibrated for $5-10 \mathrm{~ns}$ with a time step of 0.5 fs to the average temperature at which $k$ is measured. The distribution of atomic species, lattice spacing, and potential energy per atom are measured along the $z$ axis.
The system is further equilibrated at constant NVEnumber of atoms, volume, and energy-for $0.5-1 \mathrm{~ns}$. The last $10^{4}$ steps are spaced at 1.0 fs to record instantaneous atomic velocities which are later processed to determine the vibrational spectrum. Finally, nonequilibrium $\mathrm{MD}$ is employed to determine $k_{\mathrm{ZnTe}}$ with a heat source and sink, as shown in Fig. 1(b), and the non-thermostatted region is integrated using NVE with a time step of $0.5 \mathrm{fs}$. The conductivity $k=J(\mathrm{~d} T / \mathrm{d} z)^{-1}$, where $J$ denotes the heat flux and the gradient $d T / d z$ is determined through a linear fit to the temperature profile that is averaged over $40 \mathrm{~ns}$. The standard deviations of the temporal and spatial measurements are used as uncertainty measures for all the disclosed parameters. The time steps used for these simulations are conservative since, based on the vibrational spectrum presented later, a larger time step may be suitable. During deposition, the velocity of the deposited atoms should also be considered when optimizing the time step. Further details of the simulation methodology and the influence of the size of the simulation box are provided in supplementary material A and B.

$\mathrm{Zn}, \mathrm{Cd}$, and $\mathrm{Te}$ in $\mathrm{ZnTe}$ and CdTe have average potential energies of $\sim 2 \mathrm{eV}$ per atom. Since the deposited atoms have an incident energy of 5-6eV, due to momentum transfer, the energetic $\mathrm{Zn}$ and Te atoms sputter the surface atoms upon incidence. ${ }^{24}$ Ejected atoms from the substrate are evident in the deposition video included in the supplementary material. These ejected atoms are either readsorbed on the surface or deflected away from it. The periodic boundaries in the $x$ and $y$ directions and a reflector on the upper $z$-boundary prevent overall loss of ejected atoms. Hence, all the atoms are eventually reincorporated into the film.

This sputtering and reincorporation process explains the slightly asymmetric mixing profile across the interface in supplementary material $\mathrm{C}$ and the atomic mixing profiles in Figs. 2(a2) and 2(b2). It is evident that the atomic species contained in each substrate layer remain intact, except at the interface and within two preceding monolayers. In contrast, several monolayers extending from the interface into the film contain atoms that were previously part of the substrate but have now become mixed with the deposited material. The ZnTe homojunctions also exhibit this asymmetric mixing profile across their interface, which cannot be explained by the diffusivity of $\mathrm{Zn}$ and Te in $\mathrm{ZnTe}$ alone. The mixed atomic species are mainly $\mathrm{Zn}$ or $\mathrm{Cd}$, which is unsurprising since the substrate is either $\mathrm{Zn}$ terminated in $\mathrm{ZnTe}$ or $\mathrm{Cd}$ 

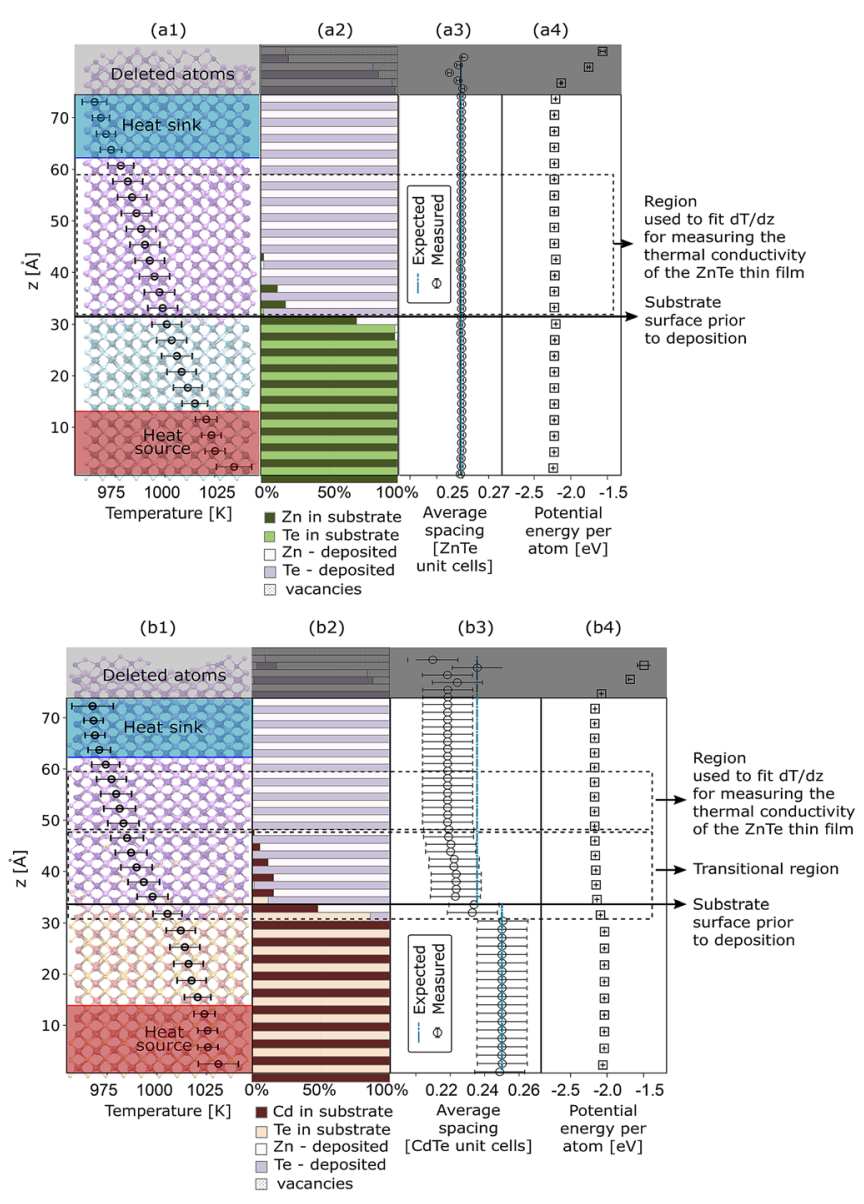

FIG. 2. (a) Homoepitaxially grown ZnTe structure. (b) Heteroepitaxially grown $\mathrm{ZnTe}-\mathrm{CdTe}$ structure. (a1) and (b1) show temperature profiles overlaid on the two-dimensional projections of the atomic coordinates. Each data point is obtained by time averaging over $40 \mathrm{~ns}$ and spatially averaging over two atomic layers that contain a total of 100 atoms. The results in (a2)-(b2) to (a4)-(b4) are evaluated post-deposition and after equilibrating the system for $10 \mathrm{~ns}$ at $1000 \mathrm{~K}$. (a2) and (b2) show the atomic species distribution. (a3) and (b3) illustrate the lattice spacing along the z-axis. At $1000 \mathrm{~K}$, the unit cells of ZnTe and CdTe are $6.150 \AA$ and $6.520 \AA$, respectively. The expected value in the figure is what the lattice spacing would have been if the film and the substrate were unstrained and did not mix during deposition. (a4) and (b4) present the potential energy profile.

terminated in CdTe. Furthermore, both ${ }_{30} \mathrm{Zn}$ and ${ }_{48} \mathrm{Cd}$ are lighter than ${ }_{52} \mathrm{Te}$, which increases their sputtering yield. ${ }^{14}$

Due to the slight mixing during deposition, both the temperature profile along the $\mathrm{ZnTe}-\mathrm{CdTe}$ heterostructure [Figs. 2(b1) and 2(b2)] and the lattice spacing along the $z$ direction [Fig. 2(b3)] exhibit a transition across the interface. Regions free of $\mathrm{Cd}$ atoms display uniform lattice spacing and potential energy per atomic layer. Interfacial mixing also occurs in the ZnTe-ZnTe homostructure [Fig. 2(a2)]. However, since identical atoms are being substituted for in this case, their mixing does not alter the atomic layer spacing [Fig. 2(a3)], potential energy per atomic layer [Fig. 2(a4)], or the temperature profile [Fig. 2(a1)].

To reduce the influence of $\mathrm{Cd}$ impurities and isolate the role of strain caused by heteroepitaxy, the values of $k$ for the deposited $\mathrm{ZnTe}$ films are evaluated over regions that are essentially $\mathrm{Cd}$ free. The selected region in the thin film for calculating $k_{\mathrm{ZnTe}}$, identified and marked in Fig. 2(b), contains a maximum of only two $\mathrm{Cd}$ atoms for any deposition case.
There is no clear correspondence between the insignificant $\mathrm{Cd}$ impurity in these regions and the value of $k_{\mathrm{ZnTe}}$.

The simulations can be summarized as follows. We use $\mathrm{Cd}_{1-q} \mathrm{Zn}_{q} \mathrm{Te}$ as the substrate and evaluate heat transfer for two extreme cases when $q=0$ and $q=1$. The corresponding change in the relative conductivity $k / k_{o}$, where the subscript $o$ refers to the unstrained value, decreases from 1 (unstrained) to $\sim 0.6$ (strained). Although not explicitly evaluated here, in principle, this value can be tuned by changing $q$ between 0 and 1. For $q=0, \mathrm{ZnTe}$ exhibits $\sim 7 \%$ uniaxial out-of-plane compressive strain and $\sim 6 \%$ biaxial in-plane tensile strain, which leads to an overall unit cell volumetric increase in the heteroepitaxial ZnTe films, see supplementary material $\mathrm{D}$ for the values.

Experimentally, ZnTe layers grown at $573 \mathrm{~K}$ on CdTe have been found to be fully strained up to $\approx 16 \AA \AA^{25}$ Beyond this thickness, the ZnTe film gradually relaxes and becomes fully relaxed at $\sim 300 \AA$. This value for the critical thickness varies in the literature and depends strongly on the growth conditions. ${ }^{26}$ While more systematic simulations are required to evaluate the applicability and the limitations of simulating strain and strain-relaxation using molecular dynamic simulations, our results based on strain induced by heteroepitaxy are nonetheless applicable when strain relaxation does not occur.

Figure 3(a) compares the relative volumetric change $V / V_{o}$ to $k / k_{o}$. These results are in excellent agreement with previous MD studies despite the differences in the constituent atoms and the method of strain implementation, i.e., strain-layer epitaxy for the $\mathrm{Cd}_{1-q} \mathrm{Zn}_{q}$ Te system vs. uniform rescaling of the simulation domain and the corresponding atomic coordinates for an $\mathrm{Ar}$ system. ${ }^{27} \mathrm{Li}$ et al. also observe similar trends for $k$ under anisotropic strain. ${ }^{13}$ The results are also consistent with experimental measurements in strained superlattices ${ }^{7,8}$
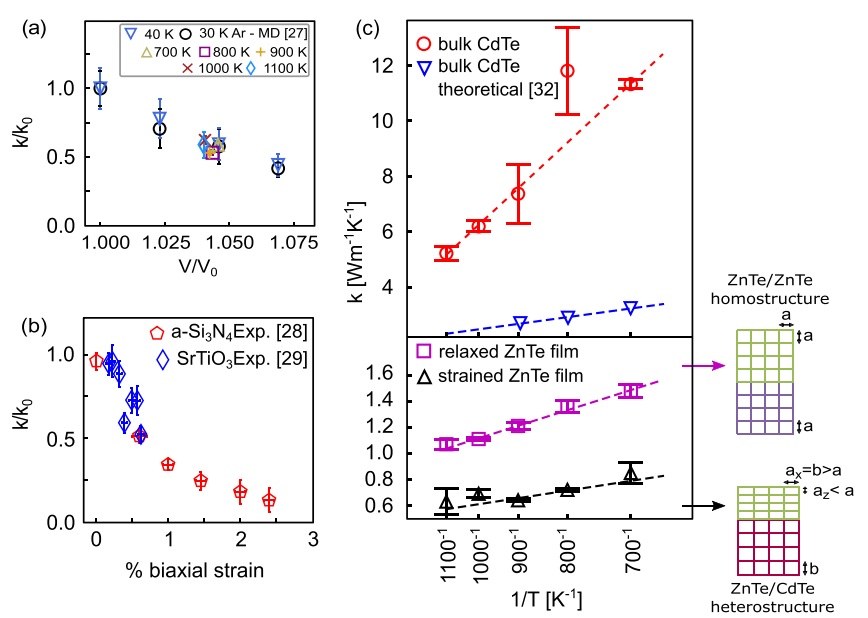

FIG. 3. (a) Thermal conductivity $k$ and average unit cell volume $V$ normalized to their unstrained values $k_{o}$ and $V_{o}$, respectively. (b) Experimental data for mechanically strained free-standing amorphous $\mathrm{Si}_{3} \mathrm{~N}_{4}$ thin films ${ }^{28}$ and for $\mathrm{SrTiO}_{3}$ thin films ${ }^{29}$ with residual strain caused by point defects. The $\mathrm{SrTiO}_{3}$ data are normalized to their maximum value (at $0.2 \%$ instead of $0.0 \%$ strain) since strain-relaxed data were not available. (c) Thermal conductivity of ZnTe films grown strain-free on ZnTe (homoepitaxy) and under strain on CdTe (heteroepitaxially). For reference, the extrapolated thermal conductivities of bulk CdTe were also evaluated through nonequilibrium MD simulations, which are presented along with their theoretically ${ }^{31}$ predicted values. The solid lines indicate the least square fits weighted to the error bars. The fitted functions are $k_{\mathrm{ZnTe} \text {,strained }}=418 / T, k_{\mathrm{ZnTe} \text {, relaxed }}=863 / T$, $k_{\mathrm{CdTe}, \text { bulk }}=11887 / T-6$, and $k_{\mathrm{CdTe}}$, ref $=1756 / T-1$. 
although direct quantitative comparison is not possible with available data. Previous experiments reveal that increasing biaxial tensile strain reduces the thermal conductivity of amorphous thin films, ${ }^{28}$ as shown in Fig. 3(b) for a $50 \mathrm{~nm}$ freestanding amorphous silicon nitride film. Furthermore, strain induced by point defects in $\mathrm{SrTiO}_{3}$ thin films also shows a reciprocal relationship between $k$ and in-plane tensile strain. ${ }^{29}$

Our simulations were designed to systematically identify the role of heteroepitaxial strain in the thermal conductivity of the deposited ZnTe thin films. Hence, in the simulations, the sole role of the CdTe substrate during $\mathrm{Zn}$ and Te deposition is to act as a template that influences how the deposited $\mathrm{Zn}$ and Te atoms are adsorbed and crystalized. In an experiment, the substrate would be of the order of microns or more. However, to reproduce the role of such a substrate during lengthy simulations of material vapor deposition, it is common to only use a few unit cells while keeping the bottom atomic layer fixed. ${ }^{11,20}$ Thus, while the CdTe film mimics a substrate used during heteroepitaxy, because of its minuscule thickness, its properties do not reflect those of an actual substrate. The thermal conductivity of bulk $\mathrm{CdTe}$, which better represents the properties of a CdTe substrate, was analyzed instead. Fifteen simulations were done at five temperatures, i.e., at 700, 800, 900, 1000, and $1100 \mathrm{~K}$, with three different simulation cell lengths for each temperature $\left[4 \times 4 \times 46,4 \times 4 \times 118\right.$, and $4 \times 4 \times 298$ (unit cells $\left.\left.{ }^{3}\right)\right]$. The bulk thermal conductivity was deduced by extrapolating $k_{\mathrm{CdTe}}$ as $L_{z} \rightarrow \infty$. The simulation details are similar to those described for bulk ZnTe in supplementary material A.

High temperature measurements of thermal conductivity are unavailable in the literature for comparison with our simulation results. The most relevant data are from a theoretical model which uses equations of phonon radiative transfer and a modification of the acoustic phonon dispersion and group phonon velocities. This model is in excellent agreement with experimental measurements taken between $100 \mathrm{~K}$ and $300 \mathrm{~K}$ and also predicts the high temperature thermal conductivity of CdTe.

Because the simulations correspond to a perfect single crystalline material while in practice the material is not defect and impurity free, MD simulations of thermal conductivity typically provide higher values than those measured experimentally. $^{22,30}$ The theoretical model accounts for impurity and dislocation scatterings which are not included in the MD simulations. ${ }^{31}$ The SW potential function used in this work may also contribute to the larger value of $k_{\mathrm{CdTe}}$ although the extent of this contribution is unknown.

From classical heat transfer, the necessary condition for thermal rectification is that $k(T, \mathbf{r})$ is a nonseparable function of both space $\mathbf{r}$ and $T .{ }^{32}$ Consider a hypothetical multilayered structure consisting of $n$ materials, where $k_{\mathrm{j}}=c_{\mathrm{j}}$ and $c_{\mathrm{j}}$ is constant for $j=1$ to $n$. Here, $k(T, \mathbf{r})=k(\mathbf{r}) k(T)$, where $k(T)$ $=1$, and so, the overall thermal conductivity is a separable function of $\mathbf{r}$ and $T$. In this case, thermal rectification cannot occur. Conversely, rectification is possible when each material segment exhibits a different $\mathrm{d} k / \mathrm{d} T$ response from its neighboring segment in the composite material. ${ }^{32}$ In this case, the functional form of $\mathrm{d} k / \mathrm{d} T$ influences the effectiveness of a thermal rectifier. ${ }^{33}$

Based on the $k(T)$ data available for bulk ZnTe and CdTe, ${ }^{18}$ the necessary condition for thermal rectification is met in a bi-segment ZnTe-CdTe structure. Here, we show that strain changes the value of $(\mathrm{d} k / \mathrm{d} T)_{\mathrm{ZnTe}}$. Linear fits to $k$ vs. $1 / T$ are shown in Fig. 3(c). Above $\theta_{\mathrm{D}}, k \propto 1 / T,{ }^{34}$ where the proportionality constant in the $\mathrm{k}-\mathrm{T}$ relation depends on the lattice constant $a$ of the material. The heteroepitaxy induced strain changes $a$, altering $\mathrm{d} k / \mathrm{d} T$. This suggests that thermal rectification in a $\mathrm{CdTe}-\mathrm{ZnTe}_{r}$ structure is different from rectification in a CdTe-ZnTe ${ }_{s}$ structure, where the subscripts $r$ and $s$ denote the relaxed and strained states, i.e., strain can change the extent of thermal rectification. The extent of rectification will also depend on the average temperature and the magnitude of the temperature gradient, i.e., $|\partial T / \partial z|$. As $|\partial T / \partial z|$ decreases, rectification and therefore relative changes in rectification become negligible. ${ }^{33}$

The vibrational density of state spectra for the ZnTe films grown on both lattice matched and lattice mismatched substrates are presented in Fig. 4. Since the system is finite in the $\pm z$ direction, the local vibrational spectrum depends on the local spatial displacement from the material boundaries. $^{35}$ To better isolate the effect of strain, identical regions identified to produce the linear $\mathrm{d} T / \mathrm{d} z$ fits, highlighted in Fig. 2(b), are compared. The vibrational spectrum is computed by ${ }^{36}$

$$
I(\omega)=\sum_{n=0}^{N} m_{n} \int e^{i \omega t}\left\langle\mathbf{v}_{n}(t+\tau) \mathbf{v}_{n}(\tau)\right\rangle_{\tau} d t
$$

and is normalized to its maximum intensity. The \langle\rangle brackets indicate the averaging of the correlation function over trajectories of different starting times $\tau, \omega$ is the angular frequency, $N$ is the total number of atoms, and $m$ is the mass of the nth atom. Additional details on the implementation are provided in supplementary material A. The strained ZnTe overlayer exhibits the red shift shown in Fig. 4, illustrating softening of the vibrational modes, which is consistent with the reduced $k^{37}$ and the unit cell expansion in the overlayer. ${ }^{38}$ We contemplate that as a unit cell expands, the Brillouin zone boundaries move inward and cut off higher phonon frequencies. Consequently, $\theta_{D}$ decreases. Since $k \propto \theta_{D}^{3}$, the conductivity also declines. ${ }^{39}$

In summary, we employ MD simulations to grow epitaxial ZnTe films on both lattice matched (ZnTe) and lattice mismatched (CdTe) substrates. The simulated deposition reveals the interfacial bond straining at the ZnTe-CdTe junction, which propagates into the ZnTe thin film during growth. The strained films undergo $\sim 6 \%$ biaxial in-plane

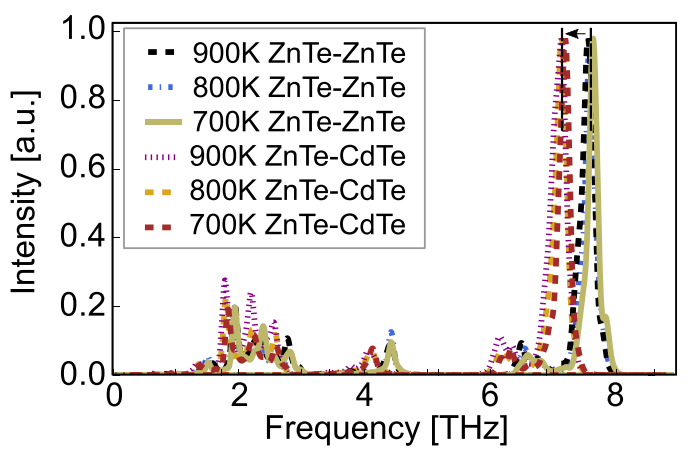

FIG. 4. Normalized vibrational density of state spectra for the ZnTe films epitaxially grown on $\mathrm{ZnTe}$ and the heteroepitaxial $\mathrm{ZnTe}$ films grown on CdTe. 
tensile strain and $\sim 7 \%$ uniaxial out-of-plane compressive strain, and so, $k$ reduces to about $60 \%$ of its unstrained value. While we use binary values for $q$ of 0 and 1 in the $\mathrm{Cd}_{1-q} \mathrm{Zn}_{q}$ Te substrate, in principle, other fractional values are possible to tune thermal conductivity. A different substrate with a lattice constant smaller than that of ZnTe can be considered to observe other trends in strain and $k$, i.e., in-plane compressive strain and out-of-plane tensile strain, which should increase $k$ of the strained $\mathrm{ZnTe}$ film. Nonequilibrium MD is used to evaluate $k$ in the temperature range of $700 \mathrm{~K}-1100 \mathrm{~K}$. The different slopes of the linear fits made to $k v s$. 1/T for the strained and unstrained $\mathrm{ZnTe}$ films suggest that strain induced by heteroepitaxy can be used to alter thermal rectification. Since thermal rectification depends on $|\partial T / \partial z|$ and decreases with decreasing $|\partial T / \partial z|$, we acknowledge that practical applications may be limited. However, the $40 \%$ reduction in thermal conductivity is significant in the context of thermoelectric devices, where strain can both decrease $k$ and enhance the mobility of charge carriers ${ }^{40-43}$ improving device performance.

See supplementary material for the details of the simulation and data analysis (A), sensitivity of thermal conductivity to the periodic dimensions of the simulation box and the simulated time length (B), molecular dynamic simulations of the deposition and growth of $\mathrm{ZnTe}(\mathrm{C})$, and axial strain in the $x$, $y$, and $z$ directions for heteroepitaxial ZnTe thin films measured at different temperatures (D). A video of the simulated thin film deposition of ZnTe on ZnTe is also included.

This work was supported by a Discovery Grant from the Natural Sciences and Engineering Research Council of Canada (NSERC Grant No. RGPIN-2014-04066). Simulations used the facilities of the Shared Hierarchical Academic Research Computing Network (SHARCNET: www.sharcnet.ca) and Compute/Calcul Canada. The authors sincerely thank Dr. Xiaowang W. Zhou from the Mechanics of Materials Department of Sandia National Laboratories in Livermore, California, and Stephen Jovanovic of the Department of Engineering Physics at McMaster University for fruitful discussions toward this work.

There are no conflicts of interest to declare.

${ }^{1}$ X. C. Tong, Advanced Materials for Thermal Management of Electronic Packaging (Springer Science \& Business Media, 2011), Vol. 30.

${ }^{2}$ D. M. Rowe, Thermoelectrics Handbook: Macro to Nano (CRC, Taylor \& Francis, 2005).

${ }^{3}$ M. Liangruksa and I. K. Puri, Appl. Phys. Lett. 102, 191907 (2013).

${ }^{4}$ H. Y. Lv, W. J. Lu, D. F. Shao, H. Y. Lu, and Y. P. Sun, J. Mater. Chem. C 4, 4538 (2016).

${ }^{5}$ T. Majdi, S. Pal, and I. K. Puri, Int. J. Therm. Sci. 117, 260 (2017).

${ }^{6}$ S. Murad and I. K. Puri, Appl. Phys. Lett. 102, 193109 (2013).

${ }^{7}$ J. Ravichandran, A. K. Yadav, R. Cheaito, P. B. Rossen, A. Soukiassian, S. J. Suresha, J. C. Duda, B. M. Foley, C.-H. Lee, Y. Zhu, A. W.
Lichtenberger, J. E. Moore, D. A. Muller, D. G. Schlom, P. E. Hopkins, A. Majumdar, R. Ramesh, and M. A. Zurbuchen, Nat. Mater. 13, 168 (2014).

${ }^{8}$ See supplementary information for Ref. 7, available at https://www.nature. com/articles/nmat3826\#supplementary-information.

${ }^{9}$ A. R. Abramson, C.-L. Tien, and A. Majumdar, J. Heat Transfer 124, 963 (2002).

${ }^{10}$ Y. Gao, Q. Liu, and B. Xu, ACS Nano 10, 5431 (2016).

${ }^{11}$ X. W. Zhou, J. J. Chavez, S. Almeida, and D. Zubia, J. Appl. Phys. 120, 45304 (2016).

${ }^{12}$ A. S. Henry and G. Chen, J. Comput. Theor. Nanosci. 5, 141 (2008).

${ }^{13}$ X. Li, K. Maute, M. L. Dunn, and Y. Ronggui, Phys. Rev. B 81, 245318 (2010).

${ }^{14}$ M. Ohring, Materials Science of Thin Films-Deposition and Structures, 2nd ed. (Academic Press, 2002).

${ }^{15}$ B. Späth, J. Fritsche, F. Säuberlich, A. Klein, and W. Jaegermann, Thin Solid Films 480-481, 204 (2005).

${ }^{16}$ M. R. H. Khan, J. Phys. D: Appl. Phys. 27, 2190 (1994).

${ }^{17}$ CRC Handbook of Chemistry and Physics, 98th ed., edited by J. R. Rumble (CRC Press, 2017).

${ }^{18}$ G. A. Slack, Phys. Rev. B 6, 3791 (1971).

${ }^{19}$ S. Plimpton, J. Comput. Phys. 117, 1 (1995).

${ }^{20}$ X. W. Zhou, D. K. Ward, J. E. Martin, F. B. Van Swol, J. L. Cruz-Campa, and D. Zubia, Phys. Rev. B 88, 085309 (2013).

${ }^{21}$ F. H. Stillinger and T. A. Weber, Phys. Rev. B 31, 5262 (1985).

${ }^{22}$ P. K. Schelling, S. R. Phillpot, and P. Keblinksi, Phys. Rev. B 65, 144306 (2002).

${ }^{23}$ X. W. Zhou, D. K. Ward, J. A. Zimmerman, J. L. Cruz-Campa, D. Zubia, J. E. Martin, and F. van Swol, J. Mech. Phys. Solids 91, 265 (2016).

${ }^{24}$ D. M. Mattox, J. Vac. Sci. Technol., A 7, 1105 (1989).

${ }^{25}$ D. Rioux, D. W. Niles, and H. Höchst, J. Appl. Phys. 73, 8381 (1993).

${ }^{26}$ S. Tatarenko, P. H. Jouneau, K. Saminadayar, and J. Eymery, J. Appl. Phys. 77, 3104 (1995).

${ }^{27}$ S. Bhowmick and V. B. Shenoy, J. Chem. Phys. 125, 164513 (2006).

${ }^{28}$ M. T. Alam, M. P. Manoharan, M. A. Haque, C. Muratore, and A. Voevodin, J. Micromech. Microeng. 22, 45001 (2012).

${ }^{29}$ S. Wiedigen, T. Kramer, M. Feuchter, I. Knorr, N. Nee, J. Hoffmann, M. Kamlah, C. A. Volkert, and C. Jooss, Appl. Phys. Lett. 100, 61904 (2012).

${ }^{30}$ Y. He, I. Savić, D. Donadio, and G. Galli, Phys. Chem. Chem. Phys. 14, 16209 (2012).

${ }^{31}$ X. Lü, J. H. Chu, and W. Z. Shen, J. Appl. Phys. 93, 1219 (2003).

${ }^{32}$ D. B. Go and M. Sen, J. Heat Transfer 132, 124502 (2010).

${ }^{33}$ C. Dames, J. Heat Transfer 131, 61301 (2009).

${ }^{34}$ C. Kittel, Introduction to Solid State Physics, 8th ed. (John Wiley \& Sons, United States of America, 2005).

${ }^{35}$ H. Bao, X. L. Ruan, and M. Kaviany, Phys. Rev. B: Condens. Matter Mater. Phys. 78, 125417 (2008).

${ }^{36} \mathrm{M}$. Thomas, Theoretical Modeling of Vibrational Spectra in the Liquid Phase (Springer, 2017).

${ }^{37}$ M. Hu, X. Zhang, and D. Poulikakos, Phys. Rev. B: Condens. Matter Mater. Phys. 87, 195417 (2013).

${ }^{38}$ J. M. Dickey, Phys. Rev. 188, 1407 (1969).

${ }^{39}$ D. T. Morelli and G. A. Slack, in High Thermal Conductivity Materials, edited by S. L. Shinde and J. S. Goela (Springer, 2006), pp. 37-68.

${ }^{40}$ E. Ungersboeck, S. Dhar, G. Karlowatz, V. Sverdlov, H. Kosina, and S. Selberherr, IEEE Trans. Electron Devices 54, 2183 (2007).

${ }^{41}$ P. R. Chidambaram, C. Bowen, S. Chakravarthi, C. Machala, and R. Wise, IEEE Trans. Electron Devices 53, 944 (2006).

${ }^{42}$ A. Nainani, D. Kim, T. Krishnamohan, and K. Saraswat, in International Conference on Simulation of Semiconductor Processes and Devices (IEEE, 2009), pp. 47-50.

${ }^{43} \mathrm{R}$. Sharma and A. K. Rana, in 2015 IEEE 2nd International Conference on Recent Trends in Information System (2015), pp. 475-480. 